\title{
Coverage Performance of MIMO-MRC in Heterogeneous Networks: A Stochastic Geometry Perspective
}

\author{
Mohammad G. Khoshkholgh*, Keivan Navaie ${ }^{\ddagger}$, Kang G. Shin ${ }^{\dagger}$, Victor C. M. Leung* \\ ${ }^{*}$ The University of British Columbia (m.g.khoshkholgh@gmail.com, vleung@ece.ubc.ca>) \\ ${ }^{\ddagger}$ Lancaster University (k.navaie@lancaster.ac.uk), ${ }^{\dagger}$ The University of Michigan (kgshin@umich.edu)
}

\begin{abstract}
We study the coverage performance of multiantenna (MIMO) communications with maximum ratio combining (MRC) at the receiver in heterogeneous networks (HetNets). Our main interest in on multi-stream communications when BSs do not have access to channel state information. Adopting stochastic geometry we evaluate the network-wise coverage performance of MIMO-MRC assuming maximum signal-tointerference ratio (SIR) cell association rule. Coverage analysis in MIMO-MRC HetNets is challenging due to inter-stream interference and statistical dependencies among streams' SIR values in each communication link. Using the results of stochastic geometry we then investigate this problem and obtain tractable analytical approximations for the coverage performance. We then show that our results are adequately accurate and easily computable. Our analysis sheds light on the impacts of important system parameters on the coverage performance, and provides quantitative insight on the densification in conjunction with high multiplexing gains in MIMO HetNets. We further observe that increasing multiplexing gain in high-power tier can cost a huge coverage reduction unless it is practiced with densification in femto-cell tier.
\end{abstract}

\section{INTRODUCTION}

Traffic demands of cellular networks-heavily driven by the popularity of video streaming and mobile social networkingare rapidly growing. Densification is one of the main approaches operators are advocating to smoothly deal with this unprecedented deluge of traffic [1]. Spectral efficiency is expectedly growing substantially because of small communication distance and universal frequency reuse. Much better performance will be, on the other hand, materialized by vastly exploiting multi-antenna (MIMO) techniques.

Nevertheless, the network performance of MIMO communications in conjunction with densification and heterogeneity is yet to be truly understood. One way to pave the road for such comprehensive assessment of the network can be achieved by adopting tools from stochastic geometry. It has been vastly employed for evaluation of the various performance metrics in wireless networks including heterogeneous networks (HetNets), see, e.g., [2], [3]. Work of [2] has proposed a flexible approach in modeling the network by $K$ tiers of independent Poisson point processes (PPPs) and maximum signalto-interference ratio (SIR) for purpose of cell association (CA). Authors in [4] have then used the framework of [2] for studying the coverage and rate performances of MISO HetNets. By providing ordering results, it has been shown that in some scenarios space-division multiple access (SDMA) was an inferior scheme to single user eigen-beamforming. In [5], [6] area spectral efficiency of MISO-SDMA systems has investigated assuming $\mathrm{CA}$ rule of maximum average received power. In [7] the outage performance of space-time block codes at the transmitters and optimal combining received filters have studied. Work of [8] has focused on the advantages of interference cancellation in zero-forcing based received filters in enhancing the coverage performance of cellular systems. However, the CA rules of [7], [8], [9] are simply a duplicate of the counterpart in single-antenna (SISO) systems of e.g., [3]. It is then very compelling to develop analysis based on CA rules that comprehensively encompass the traits of MIMO communications in improving multiplexing and diversity. For this reason, we here focus on maximum SIR rule.

We chiefly focus on multi-stream MIMO-MRC HetNets. Despite the practical significance of MIMO-MRC - chiefly because of its straightforward implementations, affordable computational complexities, and near to zero feedback overheads - the literature dealing with its performance in HetNets is small. This is because of inherent complexities rooting from residual interference among data streams each communication link suffers from. It was however previously studied in the literature of ad hoc communications, see. e.g., [10]. But, in comparison to cellular systems in ad hoc communications the network configuration lacks CA stage, which render inapplicability of the derived results therein for HetNets. Besides, in the literature of MIMO communications, both ad hoc and cellular systems, the coverage probability per a data stream was merely studied, while in reality coverage probability per a communication link (global coverage probability) comprising of multiple streams is the main performance metric. To bridge such gaps, we therefore provide accurate approximations on the latter metric via analysis. The derived bounds explicitly capture the impacts of important system parameters such as density of BSs and multiplexing gains. Our results, further, indicate that, in general, increasing multiplexing gains worsens the coverage performance of HetNets.

\section{System MOdel}

Consider downlink communication paradigms in heterogeneous cellular networks (HetNets) comprising of $K \geq 1$ tiers of randomly located BSs. BSs of tier $i \in \mathcal{K}$ are spatially 
distributed according to a homogenous Poisson Point Process (PPP) $\Phi_{i}$ with given spatial density $\lambda_{i} \geq 1$ [2]. For mathematical tractability we assume that the processes are mutually independent. Each tier $i$ can entirely be characterized with the parameters: spatial density of BSs $\lambda_{i}$, transmission power of BSs $P_{i}$ Watts, SIR threshold $\beta_{i} \geq 1$, number of BS's transmit antennas $N_{i}^{t}$, and finally the number of scheduled streams $S_{i} \leq \min \left\{N_{i}^{t}, N^{r}\right\} . S_{i}$ is referred to as multiplexing gain here. Also, $N^{r}$ is the number of antennas user equipments (UEs) possess. The modelled system of multi-stream data communication is considered as $S_{i}$ pipes of information as [11], [10]. UEs also randomly scattered across the network and form a PPP $\Phi_{U}$, independent of $\left\{\Phi_{i}\right\}$ s, with density $\lambda_{U} \gg \sum_{i} \lambda_{i}$. At each given time slot only one UE is served per active cell [12], [4], [7]. In the case that more that one UE is associated with a given BS time-sharing per cell is adopted for scheduling.

Note that according to Slivnayak's theorem and thanks to the stationarity of the point processes [13], [14], the spatial performance of the network can be adequately obtained from the eye of a typical $U E$ positioned at the origin. Let the typical UE be associated with BS $x_{i}$ transmitting $S_{i}$ data streams. Denote $\boldsymbol{y}_{x_{i}} \in \mathbb{C}^{N^{r} \times 1}$ as the received signal:

$$
\boldsymbol{y}_{x_{i}}=\left\|x_{i}\right\|^{-\frac{\alpha}{2}} \boldsymbol{H}_{x_{i}} \boldsymbol{s}_{x_{i}}+\sum_{j \in \mathcal{K}} \sum_{x_{j} \in \Phi_{j} / x_{0}}\left\|x_{i}\right\|^{-\frac{\alpha}{2}} \boldsymbol{H}_{x_{j}} \boldsymbol{s}_{x_{j}},
$$

where $s_{x_{i}}=\left[s_{x_{i}, 1} \ldots s_{x_{i}, S_{i}}\right]^{T} \in \mathbb{C}^{S_{i} \times 1}$, so that $s_{x_{i}, l} \sim$ $\mathcal{C N}\left(0, P_{i} / S_{i}\right)$, is the transmitted streams at BS $x_{i}, \boldsymbol{H}_{x_{i}} \in$ $\mathbb{C}^{N^{r} \times S_{i}}$ is the intended fading channel matrix between BS $x_{i}$ and the typical UE with entries independently drawn from $\mathcal{C N}(0,1)$, i.e., Rayleigh fading assumption. Transmitted signals are assumed independent. Likewise, channel matrices are independent. $\left\|x_{i}\right\|^{-\alpha}$ is the distance-dependent path-loss attenuation where $\left\|x_{i}\right\|$ denotes the Euclidian distance between BS $x_{i}$ and the origin, and $\alpha>2$ is the path-loss exponent. We further define $\check{\alpha}=2 / \alpha$. We assume that the typical UE knows $\boldsymbol{H}_{x_{i}}$ perfectly-perfect CSI at the receiver (CSIR).

We focus on the scenarios that BSs do not have access to the channel state information at the transmitter (CSIT). Thus BSs of each tier $i$ simply turn on $S_{i}$ transmit antennas and air information-bearing signals with fixed transmission power $P_{i}$ that is equally divided among the transmitted data streamsopen-loop technique [10], [11]. For the specific purpose of this paper maximum ratio combining (MRC) at the receiver is considered. Accordingly, for decoding $l_{i}$-th stream of data the typical receiver extracts $l_{i}$-th column of matrix $\boldsymbol{H}_{x_{i}}$ and multiplies its corresponding conjugate with the received vector (1). Let r.v.s $H_{x_{i}, l_{i}}^{\mathrm{mrc}}$ be chi-squared with $2 N^{r}$ DoFs, $\tilde{H}_{x_{i}, l_{i}}^{\mathrm{mrc}}$ be chi-squared with $2\left(S_{i}-1\right)$ DoFs, and $G_{x_{j}, l_{i}}^{\mathrm{mrc}}$ be chi-squared with DoF $2 S_{j}$, respectively, standing for the intending channel power gains associated with $l_{i}$-th data stream, inter-stream interference on stream $l_{i}$ caused by streams $l_{i}^{\prime} \neq l_{i}$, and inter-cell interference (ICI) caused by BSs $x_{j} \neq x_{i}$ on data stream $l_{i}$. Regarding [10] we can show that the SIR expression associated with $l_{i}$-th data stream is

$$
\operatorname{SIR}_{x_{i}, l_{i}}^{\mathrm{mrc}}=\frac{\frac{P_{i}}{S_{i}}\left\|x_{i}\right\|^{-\alpha} H_{x_{i}, l_{i}}^{\mathrm{mrc}}}{\frac{P_{i}}{S_{i}}\left\|x_{i}\right\|^{-\alpha} \tilde{H}_{x_{i}, l_{i}}^{\mathrm{mrc}}+\sum_{j \in \mathcal{K}} \sum_{x_{j} \in \Phi_{j} / x_{i}} \frac{P_{j}}{S_{j}}\left\|x_{j}\right\|^{-\alpha} G_{x_{j}, l_{i}}^{\mathrm{mrc}}} .
$$

Per each stream and across streams all fading coefficients are independent. Also, (2) is identically, but not independently, distributed across streams. The nominator and denominator of (2) are respectively represent the effective power of intended signal of stream $l_{i}$ and inter-stream interference plus ICI.

\section{Coverage Analysis}

We merely consider fixed-rate transmission (FRT) scheme, in which the transmission rate on each stream $l_{i}$ is constant, and equal to $R_{x_{i}, l_{i}}=\log \left(1+\beta_{i}\right) \mathrm{bit} / \mathrm{sec} / \mathrm{Hz}$, assuming that the typical UE is associated with $\mathrm{BS} x_{i}$. Typical UE is associated with the best BS that its weakest stream is stronger than the corresponding SIR threshold. To declare the coverage per communication link, FRT scheme mandates that at all $S_{i}$ scheduled streams the corresponding SIR values satisfy the required SIR threshold $\beta_{i} \geq 1$, i.e., the typical UE is claimed to be in coverage if set

$$
\mathcal{A}_{\text {frt }}=\left\{\exists i \in \mathcal{K}: \max _{x_{i} \in \Phi_{i} l_{i}=1, \ldots, S_{i}} \min _{x_{i}, l_{i}}^{\operatorname{mrc}} \geq \beta_{i}\right\},
$$

is nonempty. We therefore define coverage probability $o_{\mathrm{frt}}^{\mathrm{mrc}}=$ $\mathbb{P}\left\{\mathcal{A}_{\text {frt }} \neq \emptyset\right\}$. Note that exact evaluation of $o_{\mathrm{frt}}^{\mathrm{mrc}}$ is very complex mainly because of dependency of SIR values (2) across streams per each communication link as well as the inter-stream interference on each stream. We thus in the following resort to approximating the coverage probability.

Proposition 1: With MIMO-MRC and maximum SIR CA rule, the coverage performance can be approximated as

$$
o_{\mathrm{frt}}^{\operatorname{mrc}} \leq \sum_{i \in \mathcal{K}} \frac{\frac{\pi}{\tilde{C}(\alpha)}\left(\frac{P_{i}}{S_{i} \beta_{i}}\right)^{\check{\alpha}} \frac{\lambda_{i}}{S_{i}^{\check{\alpha}}}\left(\left.\sum_{r_{i}=0}^{N^{r}-1} \frac{(-1)^{r_{i}}}{r_{i} !} \frac{d^{r_{i}}}{d t^{r_{i}}} \frac{t^{-\frac{\check{\alpha}}{S_{i}}}}{\left(1+t \beta_{i}\right)^{S_{i}-1}}\right|_{t=1}\right)^{S_{i}}}{\sum_{j=1}^{K} \lambda_{j}\left(\frac{P_{j}}{S_{j}}\right)^{\check{\alpha}}\left(\frac{\Gamma\left(\frac{\check{\alpha}}{S_{i}}+S_{j}\right)}{\Gamma\left(S_{j}\right)}\right)^{S_{i}}} .
$$

Proof: See the Appendix.

(4) demonstrates impacts of many important system parameters such as deployment density, transmission power, multiplexing gain, and SIR threshold of tiers. Note that, in general the nominator and denominator of (4) are respectively corresponding to the intended communication link and ICI. On the other hand, the impact of inter-stream interference is captured by $\left(1+t \beta_{i}\right)^{S_{i}-1}$ that solely depends on SIR threshold and multiplexing gain.

Please note that evaluation of (4) is actually computationally affordable. But, it is yet possible to provide bounds excluding the evaluation of high-order derivatives as is carried out in following:

Proposition 2: Another approximation on the coverage probability of MIMO-MRC system with maximum SIR CA 
rule might be

$$
o_{\mathrm{frt}}^{\operatorname{mrc}} \approx \frac{\pi}{\tilde{C}(\alpha)} \sum_{i \in \mathcal{K}} \frac{\lambda_{i}\left(\frac{P_{i}}{S_{i}^{2} \beta_{i}}\right)^{\check{\alpha}}\left(\frac{\sum_{i=0}^{N_{i}^{r}-1} \frac{\Gamma\left(\frac{\check{\alpha}}{S_{i}}+\frac{S_{i}-1}{2}+r_{i}\right)}{\Gamma\left(\frac{\check{\alpha}}{S_{i}}+\frac{S_{i}-1}{2}\right) \Gamma\left(1+r_{i}\right)}}{\sum_{i_{i}=0}^{S_{i}-1}\left(\begin{array}{c}
S_{i}-1 \\
l_{i}
\end{array}\right) \beta_{i}^{l_{i}}}\right)^{S_{i}}}{\sum_{j \in \mathcal{K}} \lambda_{j}\left(\frac{P_{j}}{S_{j}}\right)^{\check{\alpha}}\left(\frac{\Gamma\left(\frac{\check{\alpha}}{S_{i}}+S_{j}\right)}{\Gamma\left(S_{j}\right)}\right)^{S_{i}}},
$$

Proof: To prove this claim, we apply the following heuristic approximation

$\left(1+t_{i} \beta_{i}\right)^{S_{i}-1}=\sum_{l_{i}=0}^{S_{i}-1}\left(\begin{array}{c}S_{i}-1 \\ l_{i}\end{array}\right) \beta_{i}^{l_{i}} t_{i}^{l_{i}} \approx t_{i}^{\frac{S_{i}-1}{2}} \sum_{l_{i}=0}^{S_{i}-1}\left(\begin{array}{c}S_{i}-1 \\ l_{i}\end{array}\right) \beta_{i}^{l_{i}}$.

Fig. 1. $\lambda_{1}=10^{-4} \cdot \alpha=4, N^{r}=10, P_{1}=50 \mathrm{~W}, P_{1}=10 \mathrm{~W}, \beta_{1}=2$,

Using this, equation (10) in appendix is reduced to

$=\sum_{i \in \mathcal{K}} \frac{\frac{\pi}{\tilde{C}(\alpha)}\left(\frac{P_{i}}{S_{i} \beta_{i}}\right)^{\check{\alpha}} \frac{\lambda_{i}}{S_{i}^{\check{\alpha}}}\left(\begin{array}{c}S_{i}-1 \\ l_{i}=0\end{array}\left(\begin{array}{c}S_{i}-1 \\ l_{i}\end{array}\right) \beta_{i}^{l_{i}}\right)^{-S_{i}}}{\sum_{j=1}^{K} \lambda_{j}\left(\frac{P_{j}}{S_{j}}\right)^{\check{\alpha}}\left(\frac{\Gamma\left(\frac{\check{\alpha}}{S_{i}}+S_{j}\right)}{\Gamma\left(S_{j}\right)}\right)^{S_{i}}}\left(\int_{0}^{\infty} \frac{\mathcal{L}_{\overline{\mathrm{F}}_{H_{i}^{\mathrm{mrc}}}}\left(t_{i}\right)}{t_{i}^{\frac{\check{\alpha}}{S_{i}}} \frac{S_{i}-1}{2}}\right)^{S_{i}}$.

The claimed result is then obtained recalling that $H_{i}^{\mathrm{rmc}}$ is chisquared r.v. with DoF $2 N^{r}$.

Corollary 1: Let $S_{i}=1 \forall i$ and $N^{r}=1$, thus (4) is reduced to $o_{\text {SISO }}=\frac{\pi}{C(\alpha)} \frac{\sum_{i \in \mathcal{K}} \lambda_{i}\left(\frac{P_{i}}{\beta_{i}}\right)^{\check{\alpha}}}{\sum_{j \in \mathcal{K}} \lambda_{j} P_{\dot{\alpha}}^{\check{\alpha}}}$, which coincides with the result of [2] of single-antenna (SISO) HetNets.

Corollary 2: Let $S_{i}=1 \forall i$ thus $o_{\mathrm{SIMO}}=$ $o_{\text {SISO }} \sum_{r=0}^{N^{r}-1} \frac{\Gamma(\check{\alpha}+r)}{\Gamma(\check{\alpha}) \Gamma(1+r)}$.

Note that in the case of SIMO scenario the results are actually accurate. On the other hand, by comparing SISO and SIMO cases it is easy to confirm that

$$
\frac{o_{\mathrm{SIMO}}}{o_{\mathrm{SISO}}}=\sum_{r=0}^{N^{r}-1} \frac{\Gamma(\check{\alpha}+r)}{\Gamma(\check{\alpha}) \Gamma(1+r)} .
$$

Using this result, one may show that by applying Kershaws inequality [15] we have

$\Gamma(\check{\alpha}) \frac{o_{\text {SIMO }}}{o_{\text {SISO }}} \geq \sum_{r=0}^{N^{r}-1}(r+0.5 \check{\alpha})^{\check{\alpha}-1} \approx \int_{0}^{N^{r}-1}(z+0.5 \check{\alpha})^{\check{\alpha}-1} d z$

or equivalently $\frac{o_{\text {SIMO }}^{\mathrm{zf}}}{o_{\text {SISO }}} \propto\left(N^{r}\right)^{\check{\alpha}}$.

\section{Simulation Results}

In this section we present simulation results. For clarity we set $K=2$. The simulation results are based on Monte Carlo technique.

We study the accuracy of the analytical findings of the paper against deploying densities of the BSs in Fig. 1 and Fig. 2. In the former (the later) we fix $\lambda_{1}=10^{-4}\left(\lambda_{2}=10^{-4}\right)$ and change $\lambda_{2}\left(\lambda_{1}\right)$. As it is seen Proposition 1 provides accurate bound on the coverage probability while the accuracy of Proposition 2 is generally questionable. However, there are scenarios, see, Fig. 2 case of $S_{1}=6$ and $S_{2}=2$, that Proposition 2 is also accurate.

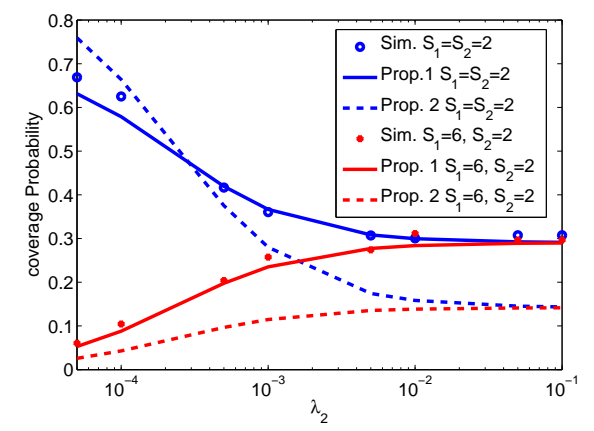

and $\beta_{2}=5$.

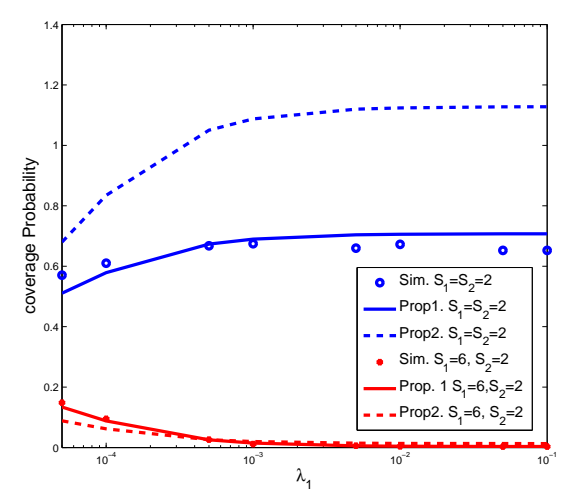

Fig. 2. $\quad \lambda_{2}=10^{-4}$. $\alpha=4, N^{r}=10, P_{1}=50 \mathrm{~W}, P_{1}=10 \mathrm{~W}, \beta_{1}=2$, and $\beta_{2}=5$.

Moreover, both of these illustrations highlight many important trends showing the impacts of multiplexing gains and densifications on the coverage performance.

First, as Fig. 1 reveals when $\lambda_{1}$ is fixed (the density of high-power BSs in tier 1) by increasing $\lambda_{2}$ smaller coverage will follow if $S_{1}=S_{2}$. In the contrary, Fig. 2 indicates that when $\lambda_{2}$ is kept fixed (density of low-power BSs in tier 2) by increasing $\lambda_{1}$ higher coverage performance is resulted again when $S_{1}=S_{2}$. In fact, for the cases that the multiplexing gains are the same across the tiers, the coverage probability could decrease/increase depends upon the tier that the densification is practiced in. The findings of these illustrations indicate that for such cases it is better to densify the tier with higher transmission power.

Second, on the other hand, Fig. 1 shows that for fixed $\lambda_{1}$, increasing $\lambda_{2}$ is beneficial and renders higher coverage performance when $S_{1}=6$ and $S_{2}=2$. Fig. 2 further highlights that when $S_{1}=6$ and $S_{2}=2$ and $\lambda_{2}$ is fixed, increasing $\lambda_{1}$ extremely exacerbates the coverage probability. Consequently, in scenarios that multiplexing gains are not the same it is better to densify the tier corresponding to low-power and low multiplexing gain.

Third, for high values of $\lambda_{2}$ Fig. 1 indicates that both scenarios of $S_{1}=6, S_{2}=2$ and $S_{1}=S_{2}=2$ perform the same. While, Fig. 2 indicates that for high values of $\lambda_{1}$ there is a huge gap between the coverage probability of regime $S_{1}=6$, 
$S_{2}=2$ and coverage probability of regime $S_{1}=S_{2}=2$. In the other words, when the network is ultra-dense in low-power tier, it is possible to increase the multiplexing gain of highpower tier without worrying about the coverage performance.

In summary, the above observations suggest that increasing the density of low power BSs (tier two) should be interpreted as a welcome for the growth of the multiplexing gains of tier 1 without damaging the coverage performance. Moreover, if we are allowed to practice densification in tier 1 , it could render higher performance provided that the similar multiplexing gains are set across the all tiers.

According to the results of both Fig. 1 and Fig. 2 we observe that increasing the density of low power BSs of tier 2 yields a much profound impact on the coverage probability than does tier 1. For example, 10 fold densification of tier 2 (tier 1) changes the coverage performance by more than $30 \%(10 \%)$. This is actually very important from practical viewpoints because installing more low-power BSs is more economically feasible than increasing the density of highpower BSs of tier 1. Finally, both of these figures confirm that for large values of $\lambda_{1}$ as well as $\lambda_{2}$ the coverage probability is stable and does not responde to densities, which is known as scale invariancy phenomenon in the literature [2].

\section{CONCLUSIONS}

We studied the coverage performance of multi-antenna (MIMO) communications with multi-stream maximum ratio combining (MRC) at the receiver in heterogenous networks (HetNets) when BSs did not have access to channel state information. We utilized powerful tools of stochastic geometry and PPP to comprehensively evaluate the network-wise coverage performance of MIMO-MRC when the cell association rule was maximum signal-to-interference ratio (SIR). Our analysis provided accurate, and easy-to-use bound of the coverage performance. Combined with simulations, it further demonstrated various important aspects of denseness and high multiplexing gains in HetNets. It was observed that increasing multiplexing gains could severely damage the coverage probability unless it practiced in high-power, low density tier in conjunction with densified low-power tier.

\section{REFERENCES}

[1] J. G. Andrews et al., "What will 5G be?" IEEE JSAC, vol. 32, no. 6, pp. 1065-1082, Jun. 2014

[2] H. S. Dhillon et al., "Modeling and analysis of $k$-tier downlink heterogeneous cellular network," IEEE JSAC, vol. 30, no. 3, pp. 550-560, Apr. 2012.

[3] H. S. Jo et al., "Heterogeneous cellular networks with flexible cell association: A compehensive downlink SINR analysis," IEEE Trans. Wireless Comm., vol. 11, no. 10, pp. 3484-3495, Oct. 2012

[4] H. S. Dhillon et al., "Downlink MIMO hetnets: Modeling, ordering results and performance analysis," IEEE Trans. Wireless. Comm., vol. 12, no. 10 , pp. 5208-5222, Oct. 2012.

[5] C. Li et al., "Success probability and area spectral efficiency in multiuser MIMO HetNets," submitted to IEEE Trans. Comm., 2015.

[6] A. K. Gupta et al., "Downlink multi-antenna heterogeneous cellular network with load balancing," IEEE Trans. Comm., vol. 62, no. 11, pp. 4052-4067, Nov. 2014.

[7] R. Tanbourgi et al., "Analysis of joint transmit-receive diversity in downlink mimo heterogeneous cellular networks," to appear in IEEE Trans. Wireless. Comm., 2015.
[8] S. T. Veetil, "Performance of pzf and mmse receivers in cellular networks with multi-user spatial multiplexing," IEEE Trans. Wireless Comm., vol. 14, no. 9, pp. 4867-34 878, Sep. 2015.

[9] C. Li et al., "Analysis of area spectral efficiency and link reliability in multiuser MIMO HetNets," in Proc. IEEE Int. Conf. Commun. (ICC), Jun. 2015.

[10] R. H. Y. Louie et al., "Open-loop spatial multiplexing and diversity communications in ad hoc networks," IEEE Trans. Inf. Theory, vol. 57, no. 1, pp. 317-344, Jan. 2011.

[11] R. Vaze and R. W. H. Jr., "Transmission capacity of ad-hoc networks with multiple antennas using transmit stream adaptation and interference cancellation," IEEE Trans. Inf. Theory, vol. 58, no. 2, pp. 780-792, Feb. 2012.

[12] V. Chandrasekhar et al., "Coverage in multi-antenna two-tier networks," IEEE Trans. Wireless. Comm., vol. 8, no. 10, pp. 5314-5327, Oct. 2009.

[13] J. F. C. Kingman, Poisson Processes. Oxford University Press, 1993.

[14] M. Haenggi and R. K. Ganti, "Interference in large wireless networks," Foundations and Trends in Networking, vol. 3, no. 2, 2008, Available at http://www.nd.edu/ mhaenggi/pubs/now.pdf.

[15] K. Huang et al., "Spatial interference cancellation for multiantenna mobile ad hoc networks," IEEE Trans. Inf. Theory, vol. 58, no. 3, pp. 1660-1676, Mrc. 2012.

[16] W. C. Ao et al., "Bounds and exact mean node degree and node isolation probability in interference-limited wireless ad hoc networks with general fading," IEEE TVT, vol. 61, no. 5, pp. 2342-2348, Jun. 2012.

[17] M. Haenggi et al., "Stochastic geometry and random graphs for the analysis and design of wireless networks," IEEE JSAC, vol. 27, no. 7, pp. 1029-1046, Sep. 2009.

\section{APPENDIX: ProOF OF PROPOSITION 1}

According to Lemma 1 in [2] and recalling that we have assumed $\beta_{i} \geq 1 \forall i$, we can write

$$
o_{\mathrm{frt}}^{\mathrm{mrc}}=\sum_{i \in \mathcal{K}} \mathbb{E} \sum_{x_{i} \in \Phi_{i}} 1\left(\min _{l=1, \ldots, S_{i}} \operatorname{SIR}_{x_{i}, l}^{\mathrm{mrc}} \geq \beta_{i}\right) .
$$

(6) is further simplified as:

$$
\begin{aligned}
& O_{\mathrm{frt}}^{\mathrm{mrc}}=\sum_{i \in \mathcal{K}} 2 \pi \lambda_{i} \int_{0}^{\infty} x_{i} \mathbb{P}\left\{\min _{l_{i}=1, \ldots, S_{i}} \operatorname{SIR}_{x_{i}, l_{i}}^{\mathrm{mrc}} \geq \beta_{i}\right\} d x_{i} \\
& =\sum_{i \in \mathcal{K}} 2 \pi \lambda_{i} \int_{0}^{\infty} x_{i} \mathbb{E}_{\left\{\Phi_{j}\right\}} \mathbb{P}\left\{\operatorname{SIR}_{x_{i}, l_{i}}^{\operatorname{mrc}} \geq \beta_{i}: \forall l_{i} \mid\left\{\Phi_{j}\right\}\right\} d x_{i} \\
& =\sum_{i \in \mathcal{K}} 2 \pi \lambda_{i} \int_{0}^{\infty} x_{i} \mathbb{E}_{\left\{\Phi_{j}\right\}} \prod_{l_{i}=1}^{S_{i}} \mathbb{P}\left\{\operatorname{SIR}_{x_{i}, l}^{\mathrm{mrc}} \geq \beta_{i} \mid\left\{\Phi_{j}\right\}\right\} d x_{i}
\end{aligned}
$$

where the first step is because of Campbell's theorem [13], and in step 4 we have used the fact that conditioned on processes $\Phi_{j}$ s SIR values across streams are statistically independent. For fix value of $x_{i}$, we now provide an expression for $\mathbb{P}\left\{\operatorname{SIR}_{x_{i}, l_{i}}^{\mathrm{mrc}} \geq \beta_{i} \mid\left\{\Phi_{j}\right\}\right\}$ as follows

$$
\begin{gathered}
\mathbb{P}\left\{\mathrm{SIR}_{x_{i}, l_{i}}^{\mathrm{mrc}} \geq \beta_{i} \mid\left\{\Phi_{j}\right\}\right\}=\mathbb{P}\left\{H_{x_{i}, l_{i}}^{\mathrm{mrc}} \geq \frac{S_{i} \beta_{i} x_{i}^{\alpha}}{P_{i}} \frac{P_{i}}{S_{i}} x_{i}^{-\alpha} \tilde{H}_{x_{i}, l_{i}}^{\mathrm{mrc}}\right. \\
\left.+\frac{S_{i} \beta_{i} x_{i}^{\alpha}}{P_{i}} \sum_{j \in \mathcal{K}} \sum_{x_{j} \in \Phi_{j} / x_{i}} \frac{P_{j}}{S_{j}}\left\|x_{j}\right\|^{-\alpha} G_{x_{j}, l_{i}}^{\mathrm{mrc}} \mid\left\{\Phi_{j}\right\}\right\} \\
=\int_{0}^{\infty} \mathcal{L}_{\overline{\mathrm{F}}_{H_{i}^{\mathrm{mrc}}}^{-1}}^{-1}\left(t_{i}\right) \prod_{j \in \mathcal{K}} \prod_{x_{j} \in \Phi_{j} / x_{i}} \mathbb{E}_{G_{x_{j}, l_{i}}^{\mathrm{mrc}}} e^{-t_{i} \beta_{i} \frac{S_{i}}{P_{i}} x_{i}^{\alpha} \frac{P_{j}}{S_{j}}\left\|x_{j}\right\|^{-\alpha} G_{x_{j}, l_{i}}^{\mathrm{mrc}}} \\
\times \mathbb{E} e^{-t_{i} \beta_{i} \tilde{H}_{x_{i}, l_{i}}^{\mathrm{mrc}} d t_{i}}
\end{gathered}
$$


$=\int_{0}^{\infty} \frac{\mathcal{L}_{\overline{\mathrm{F}}_{H_{i} \mathrm{mrc}}}^{-1}\left(t_{i}\right)}{\left(1+t_{i} \beta_{i}\right)^{S_{i}-1}} \prod_{j \in \mathcal{K}} \prod_{x_{j} \in \Phi_{j} / x_{i}} \mathbb{E}_{G_{x_{j}, l_{i}}^{\mathrm{mrc}}} e^{-t_{i} \beta_{i} \frac{S_{i}}{P_{i}} x_{i}^{\alpha} \frac{P_{j}}{S_{j}\left\|x_{j}\right\|^{\alpha}} G_{x_{j}, l_{i}}^{\mathrm{mrc}}} d t_{i}$

where $\mathcal{L}_{\overline{\mathrm{F}}_{H_{i}^{\mathrm{mrc}}}}^{-1}\left(t_{i}\right)$ is the inverse Laplace transform of $H_{i}^{\mathrm{mrc}}$ which is equal to $\mathcal{L}_{\overline{\mathrm{F}}_{H_{i}^{\operatorname{mrc}}}}^{-1}\left(t_{i}\right)=\sum_{m=0}^{N^{r}-1} \frac{1}{m !} \delta^{(m)}(t-1)$ [16], such that $\int_{0}^{\infty} e^{-t_{i} h} \mathcal{L}_{\overline{\mathrm{F}}_{H_{i} \mathrm{mrc}}}^{-1}\left(t_{i}\right) d t_{i}=e^{-h} \sum_{l=0}^{N^{r}-1} \frac{h^{l}}{l !}$, and $\delta^{(m)}(t)$ is the $m$-th derivative of Dirac delta function. Note that in (8) we have discarded index $l_{i}$ from $\mathcal{L}_{\overline{\mathrm{F}}_{H_{i}^{\mathrm{mrc}}}}^{-1}\left(t_{i}\right)$ due to the fact that $H_{x_{i}, l_{i}}^{\mathrm{mrc}}$ are identical r.v.s across streams. Substituting (8) into (7) and applying some straightforward manipulations, it is then seen that

$$
\begin{aligned}
& o_{\mathrm{frt}}^{\mathrm{mrc}}=\sum_{i \in \mathcal{K}} 2 \pi \lambda_{i} \int_{0}^{\infty} x_{i} \mathbb{E}_{\left\{\Phi_{j}\right\}} \prod_{l_{i}=1}^{S_{i}} \int_{0}^{\infty} \frac{\mathcal{L}_{\overline{\mathrm{F}}_{H_{i}^{\mathrm{mrc}}}^{-1}}^{-}\left(t_{i}\right)}{\left(1+t_{i} \beta_{i}\right)^{S_{i}-1}} \prod_{j \in \mathcal{K}} \prod_{x_{j} \in \Phi_{j} / x_{i}} \\
& \mathbb{E}_{G_{x_{j}, l_{i}}^{\operatorname{mrc}}} e^{-t_{i} \beta_{i} \frac{S_{i}}{P_{i}} x_{i}^{\alpha} \frac{P_{j}}{S_{j}}\left\|x_{j}\right\|^{-\alpha} G_{x_{j}, l_{i}}^{\mathrm{mrc}}} d t_{i} d x_{i} \\
& =\sum_{i \in \mathcal{K}} 2 \pi \lambda_{i} \int_{0}^{\infty} x_{i} d x_{i} \mathbb{E}_{\left\{\Phi_{j}\right\}} \int_{0}^{\infty} \ldots \int_{0}^{\infty} \prod_{j \in \mathcal{K}} \prod_{x_{j} \in \Phi_{j} / x_{i}} \prod_{l_{i}=1}^{S_{i}} \mathbb{E}_{G_{x_{j}, l_{i}}^{\mathrm{mrc}}} \\
& e^{-\beta_{i} \frac{S_{i}}{P_{i}} x_{i}^{\alpha} \frac{P_{j}}{S_{j}}\left\|x_{j}\right\|^{-\alpha} G_{x_{j}, l_{i}}^{\mathrm{mrc}} t_{l_{i}}} \prod_{l_{i}=1}^{S_{i}} \frac{\mathcal{L}_{\overline{\mathrm{F}}_{H_{i}^{\mathrm{mrc}}}}^{-1}\left(t_{l_{i}}\right)}{\left(1+t_{l_{i}} \beta_{i}\right)^{S_{i}-1}} d t_{l_{i}} \\
& =\sum_{i \in \mathcal{K}} 2 \pi \lambda_{i} \int_{0}^{\infty} x_{i} d x_{i} \mathbb{E}_{\left\{\Phi_{j}\right\}} \int_{0}^{\infty} \ldots \int_{0}^{\infty} \prod_{j \in \mathcal{K}} \prod_{x_{j} \in \Phi_{j} / x_{i}} \mathbb{E}_{G_{x_{j}}^{\mathrm{mrc}}} \\
& \prod_{l_{i}=1}^{S_{i}} e^{-\beta_{i} \frac{S_{i}}{P_{i}} x_{i}^{\alpha} \frac{P_{j}}{S_{j}}\left\|x_{j}\right\|^{-\alpha} G_{x_{j}, l_{i}}^{\mathrm{mrc}} t_{l_{i}}} \prod_{l_{i}=1}^{S_{i}} \frac{\mathcal{L}_{\overline{\mathrm{F}}_{H_{i}^{\mathrm{mrc}}}}^{-1}\left(t_{l_{i}}\right)}{\left(1+t_{l_{i}} \beta_{i}\right)^{S_{i}-1}} d t_{l_{i}},
\end{aligned}
$$

as r.v.s $G_{x_{j}, l_{i}}^{\mathrm{mrc}}$ are i.i.d. across streams. Consequently,

$$
\begin{aligned}
& o_{\mathrm{frt}}^{\mathrm{mrc}}=\sum_{i \in \mathcal{K}} 2 \pi \lambda_{i} \int_{0}^{\infty} x_{i} \mathbb{E}_{\left\{\Phi_{j}\right\}} d x_{i} \int_{0}^{\infty} \ldots \int_{0}^{\infty} \prod_{j \in \mathcal{K}} \prod_{x_{j} \in \Phi_{j} / x_{i}} \mathbb{E}_{G_{x_{j}}^{\mathrm{mrc}}}
\end{aligned}
$$

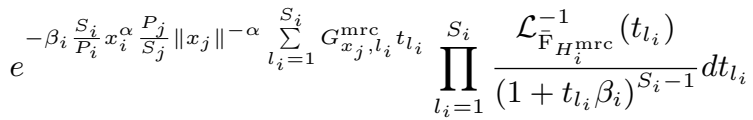

$$
\begin{aligned}
& =\sum_{i \in \mathcal{K}} 2 \pi \lambda_{i} \int_{0}^{\infty} x_{i} d x_{i} \int_{0}^{\infty} \ldots \int_{0}^{\infty} \prod_{j \in \mathcal{K}} \mathbb{E}_{\Phi_{j}} \prod_{x_{j} \in \Phi_{j} / x_{i}} \mathbb{E}_{G_{x_{j}}^{\mathrm{mrc}}}
\end{aligned}
$$

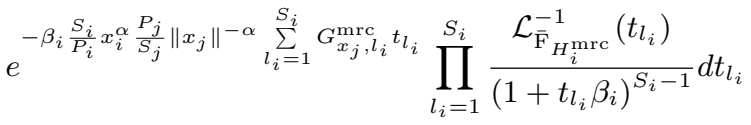

$$
\begin{aligned}
& =\sum_{i \in \mathcal{K}} 2 \pi \lambda_{i} \int_{0}^{\infty} x_{i} d x_{i} \int_{0}^{\infty} \ldots \int_{0}^{\infty} \prod_{l_{i}=1}^{S_{i}} \frac{\mathcal{L}_{\overline{\mathrm{F}}_{H_{i} \mathrm{mrc}}}^{-1}\left(t_{l_{i}}\right)}{\left(1+t_{l_{i}} \beta_{i}\right)^{S_{i}-1}} d t_{l_{i}} \\
& e^{-x_{i}^{2} \tilde{C}(\alpha)\left(\frac{S_{i} \beta_{i}}{P_{i}}\right)^{\check{\alpha}} \sum_{j=1}^{K} \lambda_{j}\left(\frac{P_{j}}{S_{j}}\right)^{\check{\alpha}} \mathbb{E}_{G_{j}} \operatorname{mrc}\left[\left(\sum_{l_{i}=1}^{S_{i}} G_{j, l_{i}}^{\mathrm{mrc}} t_{l_{i}}\right)^{\check{\alpha}}\right]},
\end{aligned}
$$

where in the last step we have applied following formula [17]:

$$
\mathbb{E}_{\Phi_{j}} \prod_{x_{j} \in \Phi_{j}} \mathbb{E}_{h_{x_{j}}} e^{-s_{j}\left\|x_{j}\right\|^{-\alpha} h_{x_{j}}}=e^{-\tilde{C}(\alpha) s_{j}^{\check{\alpha}} \mathbb{E}\left[h^{\check{\alpha}}\right]}
$$

Consequently,

$$
\begin{gathered}
O_{\mathrm{frt}}^{\mathrm{mrc}}=\sum_{i \in \mathcal{K}} 2 \pi \lambda_{i} \int_{0}^{\infty} \ldots \int_{0}^{\infty} \prod_{l_{i}=1}^{S_{i}} \frac{\mathcal{L}_{\overline{\mathrm{F}}_{H_{i}^{\mathrm{mrc}}}^{-1}}\left(t_{l_{i}}\right)}{\left(1+t_{l_{i}} \beta_{i}\right)^{S_{i}-1}} d t_{l_{i}} \\
\int_{0}^{\infty} x_{i} e^{-x_{i}^{2} \tilde{C}(\alpha)\left(\frac{S_{i} \beta_{i}}{P_{i}}\right)^{\check{\alpha}} \sum_{j=1}^{K} \lambda_{j}\left(\frac{P_{j}}{S_{j}}\right)^{\check{\alpha}} \mathbb{E}_{G_{j}^{\mathrm{mrc}}}\left[\left(\sum_{l_{i}=1}^{S_{i}} G_{j, l_{i}}^{\mathrm{mrc}} t_{l_{i}}\right)^{\check{\alpha}}\right]} d x_{i} \\
=\sum_{i \in \mathcal{K}} \frac{\pi}{\tilde{C}(\alpha)} \lambda_{i}\left(\frac{P_{i}}{S_{i} \beta_{i}}\right)^{\check{\alpha}} \int_{0}^{\infty} \ldots \int_{0}^{\infty} \prod_{l_{i}=1}^{S_{i}} \frac{\mathcal{L}_{\overline{\mathrm{F}}_{H_{i} \mathrm{mrc}}}^{-1}\left(t_{l_{i}}\right)}{\left(1+t_{l_{i}} \beta_{i}\right)^{S_{i}-1}} d t_{l_{i}} \\
\times \frac{1}{\sum_{j \in \mathcal{K}} \lambda_{j}\left(\frac{P_{j}}{S_{j}}\right)^{\check{\alpha}} \mathbb{E}_{G_{j}^{\mathrm{mrc}}}\left[\left(\sum_{l_{i}=1}^{S_{i}} G_{j, l_{i}}^{\mathrm{mrc}} t_{l_{i}}\right)^{\check{\alpha}}\right]}
\end{gathered}
$$

Unfortunately, direct evaluation of (9) seems highly untractable. Instead, we in the following resort to the arithmeticgeometric inequality for deriving an upper-bound, i.e.,

$$
\begin{gathered}
\mathbb{E}_{G_{j}^{\mathrm{mrc}}}\left[\left(\sum_{l_{i}=1}^{S_{i}} G_{j, l_{i}}^{\mathrm{mrc}} t_{l_{i}}\right)^{\check{\alpha}}\right] \geq \mathbb{E}_{G_{j}^{\mathrm{mrc}}}\left[S_{i}^{\check{\alpha}}\left(\prod_{l_{i}=1}^{S_{i}} G_{j, l_{i}}^{\mathrm{mrc}} t_{l_{i}}\right)^{\frac{\check{\alpha}}{S_{i}}}\right] \\
=S_{i}^{\check{\alpha}} \mathbb{E}_{G_{j}^{\mathrm{mrc}}}\left[\prod_{l_{i}=1}^{S_{i}}\left(G_{j, l_{i}}^{\mathrm{mrc}}\right)^{\frac{\check{\alpha}}{S_{i}}}\right]\left(\prod_{l_{i}=1}^{S_{i}} t_{l_{i}}\right)^{\frac{\check{\alpha}}{S_{i}}} .
\end{gathered}
$$

Utilizing this, (9) can be upper-bounded by

$$
\begin{aligned}
& o_{\mathrm{frt}}^{\mathrm{mrc}} \leq \sum_{i \in \mathcal{K}} \frac{\frac{\pi}{\tilde{C}(\alpha)}\left(\frac{P_{i}}{S_{i} \beta_{i}}\right)^{\check{\alpha}} \frac{\lambda_{i}}{S_{i}^{\check{\alpha}}}}{\sum_{j=1}^{K} \lambda_{j}\left(\frac{P_{j}}{S_{j}}\right)^{\check{\alpha}} \mathbb{E}_{G_{j}^{\mathrm{mrc}}} \prod_{l_{i}=1}^{S_{i}}\left(G_{j, l_{i}}^{\mathrm{rmc}}\right)^{\frac{\check{\alpha}}{S_{i}}}} \\
& \times \int_{0}^{\infty} \ldots \int_{0}^{\infty} \prod_{l_{i}=1}^{S_{i}} \frac{t_{l_{i}}^{-\frac{\check{\alpha}}{S_{i}}} \mathcal{L}_{\overline{\mathrm{F}}_{H_{i} \mathrm{mrc}}}^{-1}\left(t_{l_{i}}\right)}{\left(1+t_{l_{i}} \beta_{i}\right)^{S_{i}-1}} d t_{l_{i}}
\end{aligned}
$$

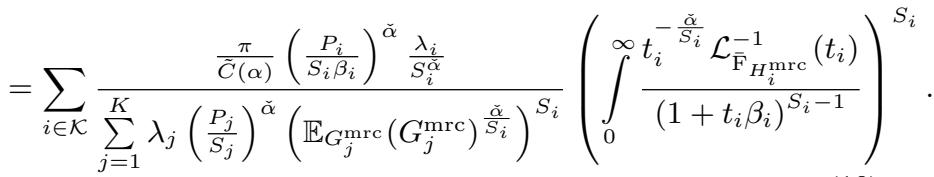

where the last step was due to the fact that r.v.s $G_{x_{j}, l_{i}}^{\mathrm{mrc}}$ are i.i.d. across streams. Note that

$$
\begin{gathered}
\int_{0}^{\infty} \frac{t_{i}^{-\frac{\check{\alpha}}{S_{i}}} \mathcal{L}_{\overline{\mathrm{F}}_{H_{i} \text { mrc }}^{-1}}^{-1}\left(t_{i}\right)}{\left(1+t_{i} \beta_{i}\right)^{S_{i}-1}}=\sum_{r_{i}=0}^{N^{r}-1} \frac{1}{r_{i} !} \int_{0}^{\infty} \delta^{\left(r_{i}\right)}\left(t_{i}-1\right) \frac{t_{i}^{-\frac{\check{\alpha}}{S_{i}}}}{\left(1+t_{i} \beta_{i}\right)^{S_{i}-1}} \\
=\left.\sum_{r_{i}=0}^{N^{r}-1} \frac{(-1)^{r_{i}}}{r_{i} !} \frac{d^{r_{i}}}{d t^{r_{i}}} \frac{t^{-\frac{\check{\alpha}}{S_{i}}}}{\left(1+t \beta_{i}\right)^{S_{i}-1}}\right|_{t=1}
\end{gathered}
$$

Also, recalling that r.v.s $G_{x_{j}, l_{i}}^{\mathrm{mrc}}$ are chi-squared with $\operatorname{DoF} 2 S_{j}$, it is straightforward to show that $\mathbb{E}_{G_{j}^{\operatorname{mrc}}}\left(G_{j}^{\mathrm{mrc}}\right)^{\frac{\check{\alpha}}{S_{i}}}=\frac{\Gamma\left(\frac{\check{\alpha}}{S_{i}}+S_{j}\right)}{\Gamma\left(S_{j}\right)}$ By substituting (11) into (10) the desired result is obtained. 